\title{
Impact of physical factors on biosphere
}

\author{
Marko S. Markov
}

Published online: 1 March 2012

(C) Springer Science+Business Media, LLC 2012

The contemporary life is characterized with an exponentially increasing dependence on a variety of physical factors. In addition to the naturally existing gravity, Earth magnetic field, and solar and space radiation, more and more man-made factors are surrounding every living creature. It is difficult to account for all the effects caused by increased background ionizing and non-ionizing radiation, ultra- and infra-sound, and temperature elevation. While global warming is a subject of scientific and political discussions, the effects of ionizing radiation has become a subject of public interest mainly after accidents such as in Chernobyl and Fukushima. In addition to this, the last three decades have placed the biosphere under the constant and increasing influence of microwave radiation from any kind of satellite, TV, radio and mobile phone communications.

The scientific community well understands the necessity to assess the impact of these factors on human life and environment. However, the publications in scientific literature are sporadic and in most cases isolated by geographic and political reasons. For example, the funding of research on mobile communications is provided mainly from the industry, which in fact immediately leads to potential bias in publications and interpretations of data.

One other serious problem is the lack of complex analysis of factors acting simultaneously. For example, Chernobyl and Fukushima nuclear accidents created increased level of background ionizing radiation, which might modulate the effects of other physical and chemical

M. S. Markov ( $\square)$

Research International, Williamsville, NY, USA

e-mail: msmarkov@aol.com factors. This is of importance for everyday life, scientific studies and clinical trials.

Driven by the necessity of a comprehensive assessment and prediction of the impact of physical factors, the UNESCO Chair of Life Science International Educational Center, Yerevan, Armenia in cooperation with ONRG/ EOARD/NFSAT hosted the International Advance Research Workshop "The Biological Impact of Electromagnetic Fields and Infra-sound at Higher Background of Ionizing Radiation".

Scientists from the USA, Japan, Armenia, Russia, Bulgaria, and Ukraine attended the meeting that took place at the Armenian resort center Tsakhkadzor, Armenia, 12-15 October 2011. The present issue of The Environmentalist is a compilation of selected paper presented at the workshop. Starting with global problems of biosphere, two very interesting papers on the impact of ionizing radiation are included in this special issue. One is actually the memoirs of the activity within and around the Chernobyl accident written by Prof. Yuri Grigoriev, who, being a participant in the radiobiological evaluations in Chernobyl, described the biological and medical problems in the 1st month after the Chernobyl accident. The second one, from the Japanese team of authors, is probably the first scientific publication for the distribution of radiation in close proximity to Fukushima accounting for the first 6 months after the disaster. A number of papers are related to biological detectors of ionizing and non-ionizing radiation as well as to natural radioprotective products.

Further, several papers treated the "global experiment" with exposing the human population and living nature to radiofrequency electromagnetic fields from mobile communications.

Following the lectures and poster sessions, round table discussion participants in the workshop agreed to issue this. 


\section{Memorandum}

Whereas life originated and developed in the presence of various physical and chemical factors,

Whereas contemporary conditions in biosphere are characterized by elevated levels of natural and man-made factors surrounding all living organisms,

Whereas global warming and climate change have enforced the impact of temperature factors on the entire biosphere,

Whereas industry development has introduced new technologies,

Whereas cellular communications, WiFi technologies and satellite investigation have increased the non-ionizing background,

Whereas nuclear accidents in Chernobyl and Fukushima significantly changed the radiation background,

We have agreed to propose:

Introduction of new standards for designing the protocols, performing the research, and reporting and publishing results from basic science and clinical studies.

This new approach MUST include detailed planning for assessment of all parameters possible to be measured or estimated in the research laboratory or/and clinical settings, such as the date, time and duration of the experiments, barometric pressure, temperature, electromagnetic and radiation background.

The scientific and medical societies should encourage their members to follow the established criteria for planning, executing of results and reporting the data. Potentially, the statistical analysis, when appropriate, should include the assessments of these data.

The scientific and medical journals should include in their instructions for preparations of manuscripts the exact list of parameters that should be fully described at submission of the manuscript and evaluated by reviewers and editors.

This special issue is one attempt to emphasize the necessity of the comprehensive evaluation of the impact of physical factors on both human life and environment. It is my pleasure to thank Prof. Sinerik Ayrapetyan for his efforts in organizing the Advance Science Workshop and further contributions in this issue. 